\title{
OPTIMAL TIMING OF VENOUS ThROMBOEMBOLIC CHEMOPROPHYLAXIS INITIATION FOLLOWING BLUNT SOLID ORGAN INJURY: META-ANALYSIS AND SYSTEMATIC REVIEW
}

Short Title (RunNing HeAd): TIMING OF VTE CHEMOPROPHYLAXIS FOR SOLID ORGAN INJURY

Patrick B. Murphy MD ${ }^{1}$, Marc de Moya MD ${ }^{1}$, Basil Karam MD ${ }^{1}$, Lauran Menard MLS ${ }^{2}$, Erik Holder $\mathrm{BS}^{2}$, Kenji Inaba $\mathrm{MD}^{3}$, Morgan Schellenberg $\mathrm{MD}^{3}$

\section{Author AfFiliations}

1

Department of Surgery, Division of Acute Care Surgery, Medical College of WISCONSIN, MiLWAUKEE, WI

2 INDIANA UNIVERSITY SCHOOL OF MEDICINE, INDIANAPOLIS, IN

3 Division of Trauma and Surgical Critical Care, LAC+USC Medical Center, UNIVERSITY OF SOUTHERN CALIFORNIA, LOS ANGELES, CA

\author{
ADDRESS FOR CORRESPONDENCE AND REPRINTS \\ Morgan Schellenberg, MD MPH FRCSC FACS \\ Division of Trauma and Surgical Critical Care \\ LAC + USC Medical Center \\ University of Southern California \\ 2051 Marengo Street \\ Inpatient Tower, C5L100 \\ Los Angeles, CA 90033 \\ Phone: (323) 409-8597 \\ Fax: (323) 441-9907 \\ E-mail: morgan.schellenberg@med.usc.edu
}




\section{Abstract}

PURPOSE: The need to prevent venous thromboembolism (VTE) following blunt solid organ injury must be balanced against the concern for exacerbation of hemorrhage. The optimal timing for initiation of VTE chemoprophylaxis is not known. The objective was to determine the safety and efficacy of early ( $\leq 48$ hours) VTE chemoprophylaxis initiation following blunt solid organ injury.

METHODS: An electronic search was performed of medical libraries for English-language studies on timing of VTE chemoprophylaxis initiation following blunt solid organ injury published from inception to April 2020. Included studies compared early ( $\leq 48$ hours) versus late ( $>48$ hours) initiation of VTE chemoprophylaxis in adults with blunt splenic, liver and/or kidney injury. Estimates were pooled using random-effects meta-analysis. Odds ratios were utilized to quantify differences in failure of nonoperative management, need for blood transfusion and rates of VTE.

RESUltS: The search identified 2,111 studies. Of these, ten studies comprising 14,675 patients were included. All studies were non-randomized and only one was prospective. The overall odds of failure of nonoperative management were no different between early and late groups, OR 1.09 (95\%CI 0.92-1.29). Similarly, there was no difference in the need for blood transfusion either during overall hospital stay, OR 0.91 (95\%CI $0.70-1.18)$, or post prophylaxis initiation, OR 1.23 (95\%CI 0.55-2.73). There were significantly lower odds of VTE when patients received early VTE chemoprophylaxis, OR 0.51 (95\%CI 0.33-0.81).

CONCLUSIONS: Patients undergoing nonoperative management for blunt solid organ injury can be safely and effectively prescribed early VTE chemoprophylaxis. This results in significantly lower VTE rates without demonstrable harm. 
KEY WORDS: trauma; deep vein thrombosis; pulmonary embolism; solid organ injury; quality improvement. 


\section{BACKGROUND}

The optimal time to initiate venous thromboembolism (VTE) chemoprophylaxis after blunt trauma is a critical clinical question without a clear, evidence-based answer. Trauma patients are known to be at particularly high risk for VTEs, especially severely injured patients who meet all three risk factors for VTE formation in Virchow's triad: hypercoagulability, venous stasis, and endothelial injury.[1] Fortunately, VTE chemoprophylaxis is effective and is associated with low (5\%) rates of VTE if initiated early ( $\leq 48$ hours). An important early study demonstrating the need for timely VTE prophylaxis initiation showed that a delay in initiation of $>4$ days is associated with a three-fold VTE risk increase.[2] It is clear that early initiation of VTE chemoprophylaxis is desirable, particularly in high-risk trauma patients. However, trauma patients often also present a risk of ongoing bleeding. Particularly among patients with blunt solid organ injuries, which are frequently managed nonoperatively, clinicians must be vigilant to monitor these patients for hemodynamic instability, worsening anemia, or ongoing transfusion requirements. Because VTE chemoprophylaxis administration may exacerbate bleeding from nonoperatively managed solid organ injuries, the desire to prevent VTEs in this high-risk population must be balanced against concern for hemorrhage.

Recently, the optimal time to initiate VTE chemoprophylaxis after blunt solid organ injury has been an area of active research focus. A number of prospective[3] and retrospective[4-12] studies have demonstrated that VTE chemoprophylaxis administration 24-48 hours after arrival to the Emergency Department (ED) is associated with reduced rates of VTE without increased need for blood transfusion or failure of nonoperative management. On this basis, we hypothesize that VTE chemoprophylaxis initiated $\leq 48$ hours of ED arrival is safe and effective at preventing VTEs without an associated increased need for blood transfusion. 
Because the existing literature is comprised of relatively small, single-center endeavors, the current study aimed to examine the optimal time for VTE chemoprophylaxis initiation after blunt solid organ injury with a meta-analysis to integrate the results of these studies into a consolidated, quantitative analysis. 


\section{METHODS}

The Preferred Reporting Items for Systematic reviews and Meta-Analyses (PRISMA) guidelines were followed for our review.[13] MEDLINE, EMBASE, Cochrane Library, CINAHL, SCOPUS, Web of Science, and ClinicalTrials.gov were searched from database inception to April 2020 to identify studies analyzing the safety of early initiation of VTE chemoprophylaxis following blunt solid organ injury managed nonoperatively. Search terms were defined by two authors (PBM, LM) and duplicated in compliance with the PRISMA minimum set of items for reporting. The search strategy for PubMed MEDLINE is detailed in Appendix 1.

All titles and abstracts were reviewed independently by two reviewers (BK, EH). Papers selected for full review were analyzed independently by two reviewers (BK, EH). The reference lists of identified studies and reviews were also examined for potentially relevant studies.

\section{Study Inclusion}

We included studies meeting the following criteria:

1) Adult ( $\geq 18$ years of age) trauma patients with any blunt solid organ injury (kidney, liver, and/or spleen) managed nonoperatively;

2) Grouped patients on time to initiation of VTE chemoprophylaxis;

3) Reported failure rates of nonoperative management (NOM) as well as need for packed red blood cells (pRBC) transfusion.

\section{Study Exclusion}

We excluded studies meeting the following criteria: 
1) Case reports $(<10$ patients);

2) Non-English language studies;

3) Conference abstracts.

4) Case Series

\section{Data Extraction}

Data were extracted and paper quality assessed by two independent reviewers (BK, EH). Conflicts were resolved by a third reviewer (PBM). A standardized data collection form was used, collecting information on study design, inclusion criteria, population demographics, injury characteristics, grade of solid organ injury, and timing of VTE prophylaxis initiation.

Clinical outcomes examined included failure of NOM, need for pRBC transfusion, number of $\mathrm{pRBC}$ transfused (both total in-hospital and post-prophylaxis initiation), and use of angioembolization.

\section{Study Quality Assessment}

Based on a systematic review of 194 tools for quality assessment we chose to use the Newcastle-Ottawa Scale to assess study quality.[14] Quality assessment was completed independently by two reviewers $(\mathrm{BK}, \mathrm{EH})$ with discussion and consensus by a third (PBM) for any disagreements. Studies were then assigned a quality rating of 'Good', 'Fair', or 'Poor' based on points in each domain.

\section{Statistical Analysis}

Meta-analysis was performed only for outcomes reported in three or more studies. Given the heterogeneity in timing of VTE prophylaxis initiation, multiple sensitivity analyses were 
performed. The most common definition of early VTE chemoprophylaxis initiation was $\leq 48$ hours, reported by 8 of the 10 included studies. Therefore, $\leq 48$ hours was chosen as the definition of early VTE chemoprophylaxis initiation for the meta-analysis. Two studies defined early VTE prophylaxis as $\leq 72$ hours rather than $\leq 48$ hours. [5,7] Because median time to first dose was not reported in these studies, these early chemoprophylaxis initiation patients were included in the early prophylaxis group of the meta-analysis. A subgroup analysis excluding these studies was also performed to investigate the impact of their inclusion. Three studies included an intermediate timing group, defined as chemoprophylaxis initiation between 48-72 hours. $[6,8,11]$ Because these patients were started on VTE chemoprophylaxis $>48 \mathrm{~h}$, they were included in the late group ( $>48 \mathrm{~h}$ ) of the meta-analysis.

Because the size of the study by Skarupa et al. was exponentially larger than the other study sample sizes, analyses were performed both with and without this study.[12] Further, Lin et al.[9] and Skarupa et al. contained duplicate patients, with both studies using years 2013-2014 of the American College of Surgeons Trauma Quality Improvement Program (ACS-TQIP) dataset. To prevent double-cohorting and because Lin et al. only examined grades III-V splenic injuries, Lin et al. was excluded from meta-analysis except for the subgroup analysis of splenic trauma.

The Mantel-Haenszel random effects model was used to calculate pooled event rates for dichotomous outcomes to calculate an odds ratio (OR) and 95\% confidence interval (CI). Heterogeneity was assessed through clinical diversity, methodological diversity, and statistical heterogeneity. Clinical diversity was assessed by comparing the cut-offs for early and late VTE prophylaxis and selection of study population between studies (distribution of solid organ injury type and grade). Statistical heterogeneity was assessed using the $\mathrm{I}^{2}$ statistic, representing the 
percentage of variability across studies attributable to differences between studies. The interpretation of $\mathrm{I}^{2}$ depends on the magnitude and direction of the effects and the strength of evidence for heterogeneity (p-value from the chi-squared test); a p-value of 0.1 was considered significant in this analysis.[15] We used accepted values for ascribing heterogeneity as follows: considerable (75-100\%); substantial (50-90\%); moderate (30-60\%) and low/not important (040\%).[15]

Publication bias was visually assessed using inverted funnel plots (Appendix 2). All analyses were performed using RevMan Version 5.3.5 (Copenhagen). The forest plots were generated to demonstrate the favored timing of VTE chemoprophylaxis initiation for each outcome. 


\section{RESUltS}

Following removal of duplicates, the literature search returned 2111 studies. After applying the inclusion criteria to the titles and abstracts, 34 articles underwent full text review (Supplemental Figure 1). Of the reviewed articles, 10 studies met final inclusion and exclusion criteria (Table 1). All studies were retrospective with the exception of one[3] and all were within the last 20 years. Two studies used the ACS-TQIP dataset for the years 2013-2014,[9,12] with Skarupa et al. examining all blunt solid organ injury within this time period and Lin et al. examining only grades III-V splenic injuries within the same time frame and dataset. Alejandro et al.[4] and Kwok et al.[8] also only examined blunt splenic trauma while Rostas et al.[11] combined both splenic and liver trauma. The remaining studies encompassed all blunt solid organ injury.

All but two were performed in the USA.[7,10] Half of the studies specifically excluded patients with head injury. The proportion of AAST grade III and higher solid organ injuries varied widely between studies (Table 2). The definition for failure of NOM was not standardized between studies but was based upon the need for either exploratory laparotomy or angioembolization at various time points (either at any time during admission or at an interval of >6-24 hours after admission) (Supplemental Table 1). The most common definition of failure of NOM was the need for laparotomy $>6$ hours after admission to hospital.

Choice of VTE chemoprophylaxis agent was also variable within and between studies and was typically at attending surgeon discretion. The most common agent was enoxaparin, dosed either once or twice daily (Supplemental Table 1). No studies reported on the use of AntiXa levels or other methods of monitoring VTE prophylaxis levels. 


\section{Failure of NOM}

Three studies reported no failures of NOM associated with either early $(\leq 48 \mathrm{~h})$ or late (>48 h) VTE chemoprophylaxis initiation, Table 3.[3,6,11] Of the remaining studies, there was no difference in failure of NOM between study groups. The overall odds of failure of NOM were 1.09 (95\% CI 0.92-1.29) (Figure 1). After excluding the largest study,[12] the odds did not change (OR 0.88, 95\% CI 0.39-1.95), (Supplement Figure 2). When including only studies examining all blunt solid organ injuries, NOM failure remained non-significant (OR 1.10, 95\%CI 0.92-1.30) (Supplement Figure 3). In a subgroup analysis of only splenic injuries, there was no difference in failure of NOM (OR 0.91, 95\% CI 0.42-1.97) (Supplement Figure 4).

\section{Bleeding}

Only six studies reported on bleeding following initiation of chemical VTE prophlyaxis, Table 3.[3,4,6,7,10,12] There was no difference in the need for blood transfusion between early or late VTE chemoprophylaxis initiation groups, OR 0.91 (95\% CI 0.70-1.18) (Figure 2). Similarly, need for any blood after receiving a dose VTE prophylaxis was no different, OR 1.23 (95\% CI 0.55-2.73) (Supplement Figure 5).

$V T E$

Two studies reported no VTE events in either the early or late VTE chemoprophylaxis initiation groups (Table 3).[4,5] In the remaining studies, there was a lower odds of VTE following blunt solid organ injury for patients receiving early chemical VTE prophylaxis, OR 0.51 (95\% CI 0.33-0.81) (Figure 3). In a sensitivity analysis excluding Skarupa et al., there was no difference in VTE events between groups, OR 0.62 (95\% CI 0.22-1.69). A subgroup analysis was performed to exclude the two studies with early VTE chemoprophylaxis initiation defined as 
$\leq 72 \mathrm{~h}[5,7]$ and there was no difference in the results with the exception of VTE, OR $0.56(95 \%$

CI 0.30-1.05).

Study Quality, Heterogeneity and Publication Bias

Overall, the study quality was Fair $(n=5)$ or Good $(n=5)$, Supplemental Table 2 . All of the studies except Schellenberg et al.[3] suffer from limitations inherent to retrospective study designs. Patient selection was well-done by all studies which identified patients based on CT findings, although exclusion criteria varied between studies, particularly in regards to the exclusion of concomitant head trauma. Time to initiation of VTE chemoprophylaxis was collected from the electronic medical administration record and no study reported on missed doses. Five studies performed adjusted analyses either with propensity scores, matching for demographic and injury characteristics, or with multivariable regression controlling for potential confounders. Except for Lin et al.'s and Skarupa et al.'s analyses of TQIP data, which includes a mandatory 30-day follow-up, loss to follow-up was not stated in any other study and resulted in lower scores in the 'Outcome' domain. Only one study performed a sample size calculation. Kwok et al. determined that 250 patients in each group would detect a $8 \%$ difference in failure of NOM.[8] Objective study heterogeneity measure by $\mathrm{I}^{2}$ was low for most analyses suggesting a low/not important risk of bias. The only significant bias was for the odds of requiring blood transfusion after receiving VTE prophylaxis, Supplementary Figure $5\left(\mathrm{I}^{2}=76 \%, \mathrm{p}=0.02\right)$. Based on visual assessment of inverted funnel plots no appreciable publication bias was detected, Appendix 2. 


\section{DISCUSSION}

Based on our systematic review and meta-analysis, early ( $\leq 48$ hours) initiation of VTE chemoprophylaxis following blunt solid organ injury is both safe and effective. Specifically, our study findings indicate that early initiation does not precipitate bleeding, with no impact on failure of NOM or need for blood transfusion. Furthermore, early initiation reduces the risk of VTE following blunt solid organ injury when compared to late ( $>48$ hours) initiation. This aligns with thromboelastography (TEG) studies demonstrating that trauma patients transition into a hypercoagulable state after approximately 24-48 hours, adding further evidence that this is the ideal time period within which to initiate VTE chemoprophylaxis.[16]

The optimal time to initiate VTE chemoprophylaxis after blunt solid organ injury has been controversial. A survey of traumatologists from Canada demonstrated large variability in timing of VTE prophylaxis initiation for these patients.[17] Additionally, the initiation time varied significantly with American Association for the Surgery of Trauma (AAST) grade of injury, with lower AAST grades associated with earlier initiation. A similar survey of AAST members on the management of splenic trauma found remarkable heterogeneity among respondents in terms of decision making around VTE chemoprophylaxis initiation, wherein providers used grade of injury, serial hemoglobin measurements, and/or a prescribed period of time after admission to guide their decision making.[18]

With competing clinical concerns about both preventing VTE formation while also avoiding provocation of bleeding from the injured viscera, the existing clinical equipoise is understandable. Similar questions have been raised in other surgical disciplines involving the care of patients at high risk for both VTE and bleeding. A review on the timing of VTE chemoprophylaxis initiation after major liver resection, for example, demonstrated a significant 
reduction in VTE without an increase in bleeding in patients with VTE chemoprophylaxis initiated $<24$ hours.[19]

The rationale for early chemical VTE prophylaxis following traumatic injury is clear. VTE is one of the most preventable causes of death following traumatic injury beyond the initial 24 hours, causing approximately $12 \%$ of deaths.[20] Both the Eastern Association for the Surgery of Trauma and the American College of Chest Physicians have acknowledged emerging evidence and the importance of VTE chemoprophylaxis in VTE prevention but have not prescribed a 'safe' initiation time.[21,22] Given the clear impetus to avoid significant risks of morbidity and mortality after VTE in this population, delineation of the optimal time frame for initiation is critically needed.

There are several limitations to this study. No randomized trials have been performed to guide the optimal time to begin chemical VTE prophylaxis following blunt solid organ injury and this hinders our interpretation of the available data. Additionally, the majority of studies captured by this study are particularly susceptible to bias due to their retrospective design, single-center nature, and/or small sample sizes. Further, the low event rate for both VTE events and failure of NOM make sound statistical analysis of differences in outcomes challenging. A major challenge in the study of VTE prophylaxis after blunt solid organ injuries is also the varying definitions of clinically important outcomes in the existing literature. This includes a lack of standard definition for the specific timing of failure of NOM and the classification of angioembolization into the operative or nonoperative group. Additional heterogeneity is added to existing literature based on different approaches to DVT screening at individual centers, with some centers screening high risk patients routinely for DVTs and other investigating symptomatic patients only. Therefore, the true incidence of VTE is unknown. Furthermore, as 
the AAST grade of solid organ injury increases, these injuries are less likely to be managed nonoperatively. Therefore, AAST grades IV-V injuries are underrepresented in the included studies and it is unclear if the study results may be safely extrapolated to these patients. Lastly, there is a potential need for risk stratification based on burden of associated injuries, especially traumatic brain injury, which has been shown to both delay VTE prophylaxis initiation and also increase the VTE risk, although this too has been challenged recently.[23] Ultimately, a large, multi-center randomized control trial will be needed to reduce bias, examine these questions in more depth, and thereby more conclusively determine the optimal time at which to initiate VTE chemoprophylaxis after blunt solid organ injury.

Blunt solid organ injury is one of the most frequent traumatic injuries and VTE is one of the most common preventable complications of trauma. Therefore, it is incumbent upon those who care for injured patients to initiate VTE chemoprophylaxis as soon as it is safe. In this systematic review and meta-analysis, early initiation of VTE chemoprophylaxis reduced VTE rates and did not increase transfusion requirements or failure rates of NOM. The best available evidence following our study supports the safe and effective initiation of VTE chemoprophylaxis $\leq 48$ hours after blunt solid organ injury in the absence of contraindications such as an associated traumatic brain injury. 


\section{COMPlianCE With ETHICAL STANDARDS}

Funding: No funding was received for this study.

Conflict of Interest: Authors Murphy, de Moya, Karam, Menard, Holder, Inaba, and Schellenberg declare that they have no conflict of interest.

Ethical approval: All procedures performed in this meta-analysis involving human participants were in accordance with the 1964 Helsinki declaration and its later amendments or comparable ethical standards. No Institutional Review Board approval was necessary due to the meta-analysis nature of the study.

Informed consent: Not applicable due to the meta-analysis nature of the study. 


\section{REFERENCES}

1. Mammen EF. Pathogenesis of Venous Thrombosis. American College of Chest Physicians 1992;102:640S-4S.

2. Nathens AB, McMurray MK, Cuschieri J, Durr EA, Moore EE, Bankey PE, Freeman B, Harbrecht BG, Johnson JL, Minei JP, McKinley BA, Moore FA, Shapiro MB, West MA, Tompkins RG, Maier RV. The Practice of Venous Thromboembolism Prophylaxis in the Major Trauma Patient. J Trauma. 2007;62(3):557-62.

3. Schellenberg M, Inaba K, Biswas S, Heindel P, Benjamin E, Strumwasser A, et al. When Is It Safe to Start VTE Prophylaxis After Blunt Solid Organ Injury? A Prospective Study from a Level I Trauma Center. World J Surg 2019;43:2797-803.

4. Alejandro K V., Acosta JA, Rodríguez PA. Bleeding Manifestations after Early Use of LowMolecular-Weight Heparins in Blunt Splenic Injuries. Am Surg 2003;69:1006-9.

5. Eberle BM, Schnüriger B, Inaba K, Cestero R, Kobayashi L, Barmparas G, et al. Thromboembolic Prophylaxis with Low-Molecular-Weight Heparin in Patients with Blunt Solid Abdominal Organ Injuries Undergoing Nonoperative Management: Current Practice and Outcomes. J Trauma 2011;70:141-6.

6. Joseph B, Pandit V, Harrison C, Lubin D, Kulvatunyou N, Zangbar B, et al. Early thromboembolic prophylaxis in patients with blunt solid abdominal organ injuries undergoing nonoperative management: Is it safe? Am J Surg 2015;209:194-8.

7. Khatsilouskaya T, Haltmeier T, Cathomas M, Eberle B, Candinas D, Schnüriger B. Thromboembolic Prophylaxis with Heparin in Patients with Blunt Solid Organ Injuries 
Undergoing Non-operative Treatment. World J Surg 2017;41:1193-200.

8. Kwok AM, Davis JW, Dirks RC, Wolfe MM, Kaups KL. Time is now: venous thromboembolism prophylaxis in blunt splenic injury. Am J Surg 2016;212:1231-6.

9. Lin B, Matsushima K, De Leon L, Piccinini A, Recinos G, Love B, et al. Early Venous Thromboembolism Prophylaxis for Isolated High-Grade Blunt Splenic Injury. J Surg Res 2019;243:340-5.

10. Murphy PB, Sothilingam N, Stewart TC, Batey B, Moffat B, Gray DK, et al. Very early initiation of chemical venous thromboembolism prophylaxis after blunt solid organ injury is safe. Can J Surg 2016;59:118-22.

11. Rostas JW, Manley J, Gonzalez RP, Brevard SB, Ahmed N, Frotan MA, et al. The safety of low molecular-weight heparin after blunt liver and spleen injuries. Am J Surg 2015;210:31-4.

12. Skarupa D, Hanna K, Zeeshan M, Madbak F, Hamidi M, Haddadin Z, et al. Is early chemical thromboprophylaxis in patients with solid organ injury a solid decision? J Trauma 2019;87:1104-12.

13. Liberati A, Altman DG, Tetzlaff J, Mulrow C, Gøtzsche PC, Ioannidis JPA, et al. The PRISMA statement for reporting systematic reviews and meta-analyses of studies that evaluate healthcare interventions: explanation and elaboration. BMJ 2009;21;339:b2700.

14. Wells G, Shea B, O'Connell D, Peterson J, Welch V, Losos M, et al. The Newcastle-Ottawa Scale (NOS) for assessing the quality of nonrandomised studies in meta-analyses. [cited 2015 Nov 29]. Available from: http://www.ohri.ca/programs/clinical_epidemiology/

15. Higgins JPT, Thompson SG, Deeks JJ, Altman DG. Measuring inconsistency in meta- 
analyses. BMJ 2003;327:557-60.

16. Chapman B, Moore E, Barnett C, Stovall R, Biffl W, Burley C, et al. Hypercoagulability following blunt solid abdominal organ injury: when to initiate anticoagulation. Am J Surg 2013;206:917-23.

17. Vogt K, Mahiri K, Murphy P, Parry N, Gray D, Moffat B, et al. The timing of initiation of venous thromboembolism prophylaxis after traumatic solid organ injury: a national survey of practice. Can J Surg 2016;59:S30.

18. Zarzaur BL, Kozar RA, Fabian TC, Coimbra R. A survey of american association for the surgery of trauma member practices in the management of blunt splenic injury. $J$ Trauma 2011;70:1026-31.

19. Baltatzis M, Low R, Stathakis P, Sheen AJ, Siriwardena AK, Jamdar S. Efficacy and safety of pharmacological venous thromboembolism prophylaxis following liver resection: a systematic review and meta-analysis. $H p b$ 2017;19:289-96.

20. Ho KM, Burrell M, Rao S, Baker R. Incidence and risk factors for fatal pulmonary embolism after major trauma: A nested cohort study. Br J Anaesth 2010;105:596-602.

21. Rogers FB, Cipolle MD, Velmahos G, Rozycki G, Luchette FA. Practice management guidelines for the prevention of venous thromboembolism in trauma patients: The EAST practice management guidelines work group. J Trauma 2002;53:142-64.

22. Kearon C, Akl EA, Comerota AJ, Prandoni P, Bounameaux H, Goldhaber SZ, et al. Antithrombotic therapy for VTE disease: Antithrombotic therapy and prevention of thrombosis, 9th ed: American College of Chest Physicians evidence-based clinical practice guidelines. Chest 
2012;141:e419S-e496S.

23. Byrne JP, Mason SA, Gomez D, Hoeft C, Subacius H, Xiong W, et al. Timing of Pharmacologic Venous Thromboembolism Prophylaxis in Severe Traumatic Brain Injury: A Propensity-Matched Cohort Study. J Am Coll Surg 2016;223:621-631.

24. Joseph B, Pandit V, Harrison C, Lubin D, Kulvatunyou N, Zangbar B, et al. Early thromboembolic prophylaxis in patients with blunt solid abdominal organ injuries undergoing nonoperative management: is it safe? Am J Surg 2015;209:194-8. 


\section{FigURE LEGENDS}

Figure 1: Odds of failure of nonoperative management after traumatic blunt solid organ injury with early $(\leq 48 \mathrm{~h})$ vs late $(>48 \mathrm{~h})$ VTE chemoprophylaxis

Figure 2: Odds of requiring a blood transfusion after traumatic blunt solid organ injury with early $(\leq 48 \mathrm{~h})$ vs late $(>48 \mathrm{~h})$ VTE chemoprophylaxis

Figure 3: Odds of venous thromboembolism after traumatic blunt solid organ injury with early $(\leq 48 \mathrm{~h}$ ) vs late $(>48 \mathrm{~h})$ VTE chemoprophylaxis 